\title{
Hexavalent Vaccinations: The Future of Routine Immunization?
}

\author{
A SHASHIDHAR \\ Department of Neonatology, St. John's Medical College Hospital, Bangalore, India. \\ shashiishere@gmail.com
}

$\mathrm{A}$ $\mathrm{s}$ the world takes rapid strides in the eradication of polio, the trivalent inactivated polio vaccine (IPV) would be the only polio vaccine available in the near future. Since the maintenance of herd immunity is important for sustaining polio eradication, this vaccine would need to be used in the early childhood immunization schedule for many years to come [1]. WHO's Strategic Advisory Group of Experts (SAGE) group's recommendation, that at least one dose of inactivated polio vaccine (IPV) along with oral polio vaccine (OPV) be administered to infants in countries currently using OPV, has been incorporated in the current national immunization schedule of India [2].

In India and other similar countries, the successful implementation of IPV depends on many aspects of vaccine delivery, including the availability of an effective and affordable vaccine. Combination (multivalent) vaccines have the potential to simplify the currently complex childhood immunization schedules, improve caregiver compliance, and reduce healthcare costs. Hexavalent vaccines containing Diphtheria (D), Tetanus (T), Pertussis (P), Hepatitis B (HBV), Haemophilus influenzae B (Hib) and the three IPV antigens have been considered logically and scientifically sound charioteers of such a strategy, and have been touted to be the ultimate combination vaccine for routine immunization [3]. Another bugbear in the success of any vaccination strategy (both public and private) is the high injection density during infancy, which cannot be ignored. To illustrate, a child gets as many as 21 injections within the first 2 years of life with the currently available combination vaccines as per Indian Academy of Pediatrics (IAP) recommendations [4]. This number comes down only slightly even if we exclude the pneumococcal vaccine. This leads to a caregiver perception that their babies receive "too many shots too soon," with vaccine hesitancy and the consequent effects including deferred doses and decreased coverage rates [5]. The use of combined vaccines offers a number of potential benefits with regard to this issue, including a reduction in the number of patient visits, reduced complications associated with multiple intramuscular injections, decreased costs of stocking and administering separate vaccines, and a reduced risk of delayed or missed vaccinations. The use of combined vaccines has been shown to improve vaccine coverage and timeliness [6].

The research paper by Chhatwal, et al. [7] published in this edition of Indian Pediatrics evaluates the efficacy of one such vaccine that is already approved in many countries, and is being used in their national schedules. The authors demonstrate very good seroconversion for all components even without a control group, and show how it can be integrated into the current immunization schedules. Moreover, the fully liquid preparations of hexavalent vaccines have distinct advantages over those which require reconstitution. Average preparation time is found to be almost half for the fully-liquid vaccine compared the non-fully-liquid vaccine. In the same study, almost all health care personnel (97.6\%) stated that they would prefer the use of the fully-liquid vaccine in their daily practice [8].

Regarding pertussis vaccination, recent reports suggest a waning of protective immunity in adolescence that might be responsible for an increase in cases of pertussis in infants under six months of age, before they are fully vaccinated [9]. In high-income countries, this waning immunity to pertussis is being addressed by additional booster immunizations through the use of DTaP to replace DT in older age groups. However, due to the higher cost, this is not considered to be a viable option for lower-middle income countries like India. WHO also observes the hypothesis that transition from wP (whole cell pertussis) to aP (acellular pertussis) vaccines may be associated with resurgence of disease. Though the licensed aP and wP vaccines have equivalent efficacy in preventing disease in the first year of life, there is a more rapid waning of protection, and possibly a reduced impact on disease transmission, with aP compared to wP vaccines. It states that a switch from $w P$ to aP vaccines for primary infant immunization should only be considered if the inclusion in the national immunization schedules of additional periodic booster or maternal immunization can be assured and sustained [10]. Also, the IAP Advisory Committee on Vaccines \& Immunization Practices 
(ACVIP) recommends that $\mathrm{DTaP}$ vaccine/combinations should preferably be avoided for the primary series and if any acellular pertussis containing vaccine is used, it must at least have three or more components in the product, which is not the case in this investigational vaccine [4]. Interestingly, no hexavalent combinations with whole cell pertussis (wP) are commercially available or in late-stage of development because of numerous technical and logistical issues, and are unlikely to be available in next few years [3]. The classic wP vaccine with thimerosal also faces a major challenge of incompatibility with IPV antigens. The production of these vaccines is technically resource-intensive, which increases production costs and therefore, many manufacturers target them as premium products. Therefore, the hexavalent vaccines that are most likely to be available now, or in the very near future are primarily intended and priced for private markets. With regard to the newer Sabin IPV, although some vaccine manufacturers have access to the Sabin strains (for OPV product), further effort and time would be needed to develop an inactivated form that is compatible for combination vaccine development [3].

There have been a few issues with the combination vaccines themselves in the past. Hexavac (Sanofi Pasteur MSD, Lyon, France), which was licensed in Europe in 2000 as a pediatric primary and booster immunization, was recommended for suspension of marketing authorization by European Medicines Agency in September 2005 because of the reduced immunization properties of the HBV component [11]. However, the newer vaccine from the same manufacturers - Hexyon or Hexaxim - has a higher HBsAg content and uses a different method for its production. This seems to have resulted in higher immunogenicity compared to hexavac [12]. A possible temporal association between first immunization with hexavac and the occurrence of sudden unexpected death was also suspected [13]. This claim was strongly refuted on further investigation. The other widely used hexavalent vaccine Infanrixhexa (GSK, Riixensart, Belgium), which contains three pertussis antigens (PT, FHA and PRN), has been demonstrated to be immunogenic, effective, safe and well tolerated in children regardless of gestational age at birth, and not significantly different from the vaccines used as comparators. The immunogenicity seems superior to Hexavac for hepatitis B until 7-9 years of age [12]. Furthermore, vaccination with this DTaP-HBV-IPV/Hib in infancy induces sustained seroprotection and immune memory against $\mathrm{HBV}$ even in 12-13 year-old adolescents [14]. The comparison of this vaccine between two schedules in Indian infants has also been recently published [15]. With regards to the current study, the immunological response with co-administration of PCV7 and rotavirus vaccine was not studied. Also, the persistence of seroprotection, especially with pertussis (having two antigens), and the effect of administration of a booster dose were not evaluated.

In conclusion, these hexavalent formulations are already a necessary component of the vaccination schedule, and would be even more pertinent in the days ahead. However, they would need to be monitored for long-term effectiveness in view of the growing concern of waning immunity against diseases such as pertussis when using acellular-pertussis vaccine, and possibly hepatitis $\mathrm{B}$ when using combination vaccines.

Funding: None; Competing interest: None stated.

\section{REFERENCES}

1. Patriarca PA, Sutter RW, Oostvogel PM. Outbreaks of paralytic poliomyelitis, 1976-1995. J Infect Dis. 1997; 175:S165-72.

2. Indian Academy of Pediatrics (IAP) Advisory Committee on Vaccines and Immunization Practices (ACVIP), Vashishtha VM, Choudhary J, Yadav S, Unni JC, Jog P, Kamath SS, et al. Introduction of inactivated poliovirus vaccine in National immunization program and polio endgame strategy. Indian Pediatr. 2016;53(Suppl 1):S65-9.

3. Mahmood K, Pelkowski S, Atherly D, Sitrin R, Donnelly JJ. Hexavalent IPV-based combination vaccines for publicsector markets of low-resource countries. Hum Vaccin Immunother. 2013;9:1894-902.

4. Vashishtha VM, Choudhury P, Kalra A, Bose A, Thacker $\mathrm{N}$, Yewale VN, et al. Indian Academy of Pediatrics (IAP) recommended immunization schedule for children aged 0 through 18 years - India, 2014 and updates on immunization. Indian Pediatr. 2014;51:785-800.

5. Meyerhoff AS, Jacobs RJ. Do too many shots due lead to missed vaccination opportunities? Does it matter? Prev Med. 2005;41:540-4.

6. Marshall GS, Happe LE, Lunacsek OE, Szymanski MD, Woods CR, Zahn M, et al. Use of combination vaccines is associated with improved coverage rates. Pediatr Infect Dis J. 2007;26:496-500.

7. Chhatwal J, Lalwani S, Vidor E. Immunogenicity and safety of a liquid hexavalent vaccine in Indian infants. Indian Pediatr. 2017;54:15-20.

8. De Coster I, Fournie X, Faure C, Ziani E, Nicolas L, Soubeyrand B, et al. Assessment of preparation time with fully-liquid versus non-fully liquid paediatric hexavalent vaccines. A time and motion study. Vaccine. 2015;33:3976-82.

9. Witt MA, Katz PH, Witt DJ. Unexpectedly limited durability of immunity following acellular pertussis vaccination in preadolescents in a North American outbreak. Clin Infect Dis. 2012;54:1730-5.

10. Pertussis vaccines: WHO position paper - September 2015. Wkly Epidemiol Rec. 2015;90:433-58.

11. European Medicines Agency. European Medicines Agency recommends suspension of Hexavac. Available from: http:/ 
/www.ema.europa.eu/ema/index.jsp? curl=pages/news and_events/news/2009/12/news_detail_000855.jsp\& $m i d=W C 0 b 01 a c 058004 d 5 c 1$. Accessed October 27, 2016.

12. Esposito S, Tagliabue C, Bosis S, Ierardi V, Gambino M, Principi N. Hexavalent vaccines for immunization in paediatric age. Clin Microbiol Infect. 2014;20(S5):76-85.

13. von Kries R, Toschke AM, Strassburger K, Kundi M, Kalies H, Nennstiel U, et al. Sudden and unexpected deaths after the administration of hexavalent vaccines (diphtheria, tetanus, pertussis, poliomyelitis, hepatitis B, Haemophilius influenzae type b): is there a signal? Eur J Pediatr.
2005; 164:61-9.

14. Behre U, Van Der Meeren O, Crasta P, Hanssens L, Mesaros N. Lasting immune memory against hepatitis B in 12-13-year-old adolescents previously vaccinated with 4 doses of hexavalent DTPa-HBV-IPV/Hib vaccine in infancy. Hum Vaccin Immunother. 2016;12:2916-20..

15. Lalwani SK, Agarkhedkar S, Sundaram B, Mahantashetti NS, Malshe N, Agarkhedkar S, et al. Immunogenicity and safety of 3-dose primary vaccination with combined DTPa$\mathrm{HBV}-\mathrm{IPV} / \mathrm{Hib}$ in Indian infants. Hum Vaccin Immunother. 2016 Sep 15:1-8. [Epub ahead of print]. 